\title{
The Implication of Cytogenetic Alterations in Pancreatic Ductal Adenocarcinoma and Intraductal Papillary Mucinous Neoplasm Identified by Fluorescence In Situ Hybridization and Their Potential Diagnostic Utility
}

\author{
Chang-Sup Lim ${ }^{1}$, Kyongok Im ${ }^{2,3}$, Dong Soon Lee ${ }^{2,3}$, Wooil Kwon ${ }^{3,4}$, Jae Ri Kim ${ }^{3,4}$, Youngmin Han ${ }^{3,4}$, Sun-Whe Kim ${ }^{3,4}$, and Jin- \\ Young Jang ${ }^{3,4}$ \\ ${ }^{1}$ Department of Surgery, SMG-SNU Boramae Medical Center, ${ }^{2}$ Department of Laboratory Medicine, Seoul National University Hospital, Seoul \\ National University College of Medicine, ${ }^{3}$ Cancer Research Institute, Seoul National University College of Medicine, and ${ }^{4}$ Department of \\ Surgery, Seoul National University Hospital, Seoul National University College of Medicine, Seoul, Korea
}

\section{See editorial on page 397.}

Background/Aims: We investigated chromosomal aberrations in patients with pancreatic ductal adenocarcinoma (PDAC) and intraductal papillary mucinous neoplasm (IPMN) by fluorescence in situ hybridization (FISH) to identify cytogenetic changes and molecular markers that may be useful for preoperative diagnosis. Methods: Tissue samples from 48 PDAC and 17 IPMN patients were investigated by FISH analysis using probes targeting chromosomes $7 \mathrm{q}$, $17 p, 18 q, 20 q$, and $21 q$ and the pericentromeric region of chromosome 18 (CEP18). Results: The PDAC samples harbored $17 p$ deletion (95.8\%), 18q deletion (83.3\%), CEP18 deletion (81.2\%), 20q gain (81.2\%), 21q deletion (77.1\%), and $7 q$ gain (70.8\%). The IPMN samples had $17 p$ deletion (94.1\%), CEP18 deletion (94.1\%), 21q deletion (70.6\%), 18q deletion (58.8\%), 20q gain (58.8\%), and $7 q$ gain (58.8\%). A significant difference in CEP18 gain was identified between the PDAC and IPMN groups ( $p=0.029)$. Detection of $17 p$ or $18 q$ deletion had the highest diagnostic accuracy (80.0\%) for PDAC. Conclusions: Chromosomal alterations were frequently identified in both PDAC and IPMN with similar patterns. CEP18 gain and $17 p$ and $18 q$ deletions might be involved in the later stages of PDAC tumorigenesis. Chromosome $17 p$ and $18 q$ deletions might be excellent diagnostic markers. (Gut Liver 2020;14:509-520)

Key Words: Carcinoma, pancreatic ductal; Pancreatic intraductal neoplasms; In situ hybridization, fluorescence;
Chromosomal aberrations

\section{INTRODUCTION}

Pancreatic ductal adenocarcinoma (PDAC) is the fourth leading cause of cancer death, and is projected to become the second leading cause of cancer-related death in the United States by 2030. ${ }^{1}$ Surgical resection remains the only curative treatment modality; however, most patients are ineligible for surgery because of advanced disease at diagnosis. ${ }^{2}$

To develop effective methods for early stage diagnosis of this deadly disease which could improve its outcomes, it is important to identify the fundamental genetic changes involved in PDAC carcinogenesis. Activation of the K-RAS oncogene and inactivation of the tumor suppressor genes CDKN2A/ INK4A, TP53, and SMAD4 were recently reported to make key contributions to PDAC carcinogenesis. ${ }^{3}$ Cytogenetic studies of PDAC have also identified numerous complex structural and numerical alterations at the subchromosomal level, and copy number gain of 3q, 5p, 7p, 8q, 11q, 12p, 17q, 19q, and 20q, and loss of 1p, 3p, 4q, 6q, 8p, 9p, 10q, 12q, 13q, 15q, 17p, $18 q, 19 p, 21 q$, and 22q, are recurrent aberrations in PDAC., However, cytogenetic studies of PDAC are often complicated by a strong desmoplastic reaction and inflammatory cells. ${ }^{6}$ Accordingly, most analyses have been of pancreatic cell lines, short-term cultures, or xenografted tumor cells; hence recorded genomic alterations could potentially have been acquired in vitro. ${ }^{7}$ Moreover, most reported chromosomal alterations in PDAC have been from the minority of patients who present without detectable metastases and are eligible for surgery. Also,

Correspondence to: Jin-Young Jang

Department of Surgery, Seoul National University College of Medicine, 101 Daehak-ro, Jongno-gu, Seoul 03080, Korea

Tel: +82-2-2072-2194, Fax: +82-2-741-2194, E-mail: jangjy4@snu.ac.kr

Received on March 14, 2019. Revised on June 18, 2019. Accepted on July 8, 2019. Published online September $20,2019$. pISSN 1976-2283 eISSN 2005-1212 https://doi.org/10.5009/gnl19087

(c) This is an Open Access article distributed under the terms of the Creative Commons Attribution Non-Commercial License (http://creativecommons.org/licenses/by-nc/4.0) which permits unrestricted non-commercial use, distribution, and reproduction in any medium, provided the original work is properly cited. 
as a substantial proportion of patients develop recurrent disease following surgery, detected genetic changes may not represent early events in carcinogenesis. ${ }^{8}$ Therefore, studies of PDAC precursor conditions, such as intraductal papillary mucinous neoplasm (IPMN) and pancreatic intraepithelial lesion (PanIN), are essential to identify early events in the process of PDAC carcinogenesis. Recently, an increase in the number of patients diagnosed with IPMN (the most common precursor lesion of PDAC) has been described, likely due to incidental discovery with new imaging techniques. However, only a few studies have reported the genetic alterations in these tumors because of difficulties obtaining tissue samples without using invasive procedures, such as surgery. ${ }^{9-13}$

It is also important for clinicians to discriminate PDAC from other benign pancreatic diseases to facilitate establishment of appropriate therapeutic plans. Endoscopic ultrasound (EUS)guided fine-needle aspiration or cytologic brushing is the current standard method for tissue acquisition and pathologic diagnosis of pancreatic lesions. However, histopathologic diagnosis is often difficult and diagnostic sensitivity is consequently unsatisfactory. ${ }^{14-16}$ Fluorescence in situ hybridization (FISH) is a clinically useful technique, as it can be conducted using limited tissue material, including smears and brushings, and is rapid and relatively accessible. ${ }^{9}$ Several studies have reported the clinical utility of FISH analysis using the commercially available probe set, UroVysion (Abbott Molecular Inc, Des Plaines, IL, USA), and the Papanicolaou Society of Cytopathology adopted FISH as a useful ancillary test to complement routine cytology. ${ }^{17-19}$ However, The UroVysion FISH analysis method was developed for diagnosis of bladder cancer using urine samples, and its probe set contains 9p21 which is directed to the $C D K$ N2A gene, and chromosome enumeration probes (CEPs) directed to chromosomes 3, 7, and 17. To increase diagnostic accuracy for PDAC, it will be essential to identify the optimal probes targeting chromosomal regions typical of this tumor type.

Most of the previous studies concerning the chromosomal alteration of PDAC and IPMN has been conducted in Western countries, and there has been only a few reports from Asian countries. ${ }^{11,12,20,21}$ In addition, previous studies did not show consistent chromosomal alteration, which might imply that there could be many genetic variations in the genesis and progression of PDAC and IPMN. We assume that the differences of chromosomal alteration between PDAC and IPMN might provide valuable information about carcinogenesis because IPMN is a well-known premalignant disease. The commonality of genetic alteration between the two diseases might be presumed to occur in the earlier stages of carcinogenesis, and the differences might be occur in the later steps. Besides, these differences might be also helpful in the preoperative diagnosis of PDAC. In this study, we investigated the chromosomal aberrations in PDAC and IPMN patient samples by FISH analysis using probes targeting chromosomes reported as frequently altered in PDAC, to identify the patterns and differences of chromosomal alteration in Korean PDAC and IPMN patients, and to investigate the optimal probe sets that may aid preoperative diagnosis of PDAC.

\section{MATERIALS AND METHODS}

\section{Patients and sample collection}

Prospectively collected tumor tissue samples from patients who underwent pancreatic resection at the Seoul National University Hospital (Seoul, Korea) from April 2015 to July 2016 were investigated. The patients had PDAC $(n=48)$ or IPMN $(n=17)$. Among those with IPMN, two, four, and five patients had mild, moderate, and severe dysplasia, respectively, while six had invasive IPMN. Invasive IPMN was defined when there as the presence of an invasive carcinoma derived from (arising in the area of) IPMN pathologically. ${ }^{22}$ All 65 patients were scheduled for surgery with a suspicion of malignancy in radiological evaluations. Preoperative histologic diagnoses using EUS-guided fineneedle aspiration were performed in 25 of 48 PDAC (52.1\%) and in 5 of 17 IPMN patients (29.4\%), and were possible to make a diagnosis of malignancy in 19 of 25 PDAC (76.0\%), and in four of five IPMN patients (80.0\%).

The demographic and pathologic characteristics of the patients, and FISH results for chromosomes 7q, 17p, 18q, 20q, and 21q, and a chromosome enumeration probe 18 (CEP18), were investigated. Pathologic staging was determined according to the seventh edition of the American Joint Committee on Cancer staging system. ${ }^{23}$ Informed consent for tissue sample collection for research purposes was obtained from individual patients preoperatively, and the study protocol, as well as ethical issues, were reviewed and approved by the Institutional Review Board at Seoul National University Hospital (IRB numbers: H-0901010-267 and H-1807-099-960). Each tumor sample was harvested immediately after surgical resection and stored in liquid nitrogen. Some of the harvested tumor sample was sent to a pathologist and confirmed by frozen section biopsy to ensure that the tumor tissue was properly harvested.

\section{Fluorescence in situ hybridization}

Tumor samples were transported to the laboratory in an icebox. For FISH examination, tissue samples were minced with a surgical scalpel and incubated in collagenase type IV (1 mg/ $\mathrm{mL}$ ) (STEMCELL Technologies, Vancouver, BC, Canada) for 20 minutes. After washing with phosphate-buffered saline, samples were filtered using $100 \mu \mathrm{m}$ cell strainers (BD Falcon, Franklin Lakes, NJ, USA) to generate a single cell suspension, followed by centrifugation for 5 minutes at 1,200 rpm. After adding 5 $\mathrm{mL}$ of $0.075 \mathrm{M} \mathrm{KCl}$ to each tube, samples were incubated for 25-30 minutes in a $37^{\circ} \mathrm{C}$ water bath. Carnoy's fixative (500 $\mu \mathrm{L}$ ) was added, and samples were incubated for 5 minutes at room temperature. Suspensions were centrifuged for 5 minutes at 1,200 rpm, and supernatants were removed. Pellets were 
resuspended in 3-5 $\mathrm{mL}$ of Carnoy's fixative and incubated for 20 minutes at room temperature, then suspensions were centrifuged for 5 minutes at 1,200 rpm and the supernatants were removed; this step was performed twice. Next, fixed cells were mixed with Carnoy's fixative and dropped onto microscope slides. Air-dried slides were pretreated with $2 \times$ standard saline citrate (SSC; $300 \mathrm{mmol} / \mathrm{L}$ sodium chloride and $30 \mathrm{mmol} / \mathrm{L}$ sodium citrate) for 30 minutes at $37^{\circ} \mathrm{C}$, and dehydrated with cold 70\%, 85\%, and 100\% ethanol for 2 minutes each. Under protection from light, FISH probes were added to the prepared slides, which were then covered with coverslips and sealed with rubber cement. FISH probes used were as follows: XL Spectrum Orange (7q22)/Spectrum Green (7q36), XL ATM Spectrum Green (11q22)/TP53 Spectrum Orange (17p13), XL MALT Break Apart Spectrum Orange/Green (18q21) (MetaSystems, Altlussheim, Germany), Vysis CEP 18 (D18Z1) Spectrum Orange (Abbott Molecular), IGH Spectrum Green (14q32.33)/MAFB Spectrum Red (20q12) (Cytocell Ltd, Cambridge, UK), and Vysis RUNX1 Spectrum Green (21q22)/RUNX1T1 Spectrum Orange (8q21) (Abbott Molecular). Probes and target DNA were simultaneously denatured at $75^{\circ} \mathrm{C}$ for 5 minutes, then slides were hybridized for $10-16$ hours at $37^{\circ} \mathrm{C}$ in a hybridizer (Dako, Glostrup, Denmark). After hybridization, slides were washed in $0.4 \times \mathrm{SSC}$ at $73^{\circ} \mathrm{C}$ for 2 minutes, and in $0.1 \%$ Nonidet $\mathrm{P}-40 / 2 \times$ SSC at room temperature for 2 minutes. Chromosomes were counterstained with $10 \mu \mathrm{L}$ of $4^{\prime}-6^{\prime}$-diamine-2-phenylindole dihydrochloride (DAPI/Antifade) (MetaSystems). Images were analyzed using a Zeiss Axioplan 2 imaging microscope (Carl Zeiss MicroImaging $\mathrm{GmbH}$, Munich, Germany) with the ISIS software (MetaSystems). Approximately 100 nuclei were scored for each probe (Fig. 1). Nuclei with ambiguous signals and cells with poor morphology were excluded from scoring. The absolute cutoff values of FISH analysis using tissue samples has not yet been established, and

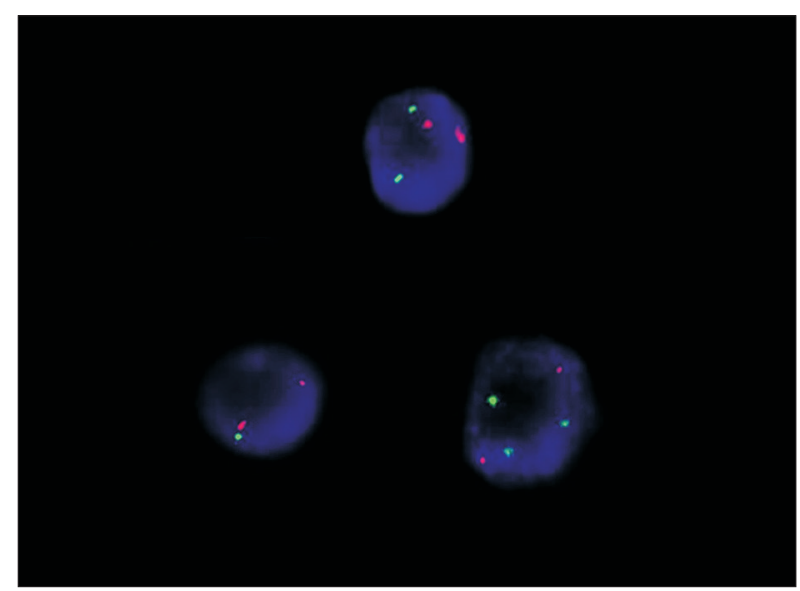

Fig. 1. Fluorescence in situ hybridization analysis of $21 \mathrm{q} 22$ using a colored probe. A normal (disomic) cell is shown with two green signals (top). Heterozygous deletion of 21q22 is shown in the cell with one green signal (bottom left), and heterozygous gain of 21q22 is indicated by three green signals (bottom right). we adopted the binomial treatment of the data to set the cutoff values because it was one of the reliable methods to calculate the cutoff values of FISH analysis in the hematological diseases. Based on FISH analysis of 18 normal pancreatic tissue samples in a preliminary study, cutoff values for the normal range for FISH analysis were calculated using the Excel 2013 (Microsoft Corp., Redmond, WA, USA) statistical function CRITBINOM $(n, p, \alpha)$ with a confidence level of 95\% (Table 1). ${ }^{24}$ When the percentage of cells containing $>2$ or $<2$ FISH signals exceeded the cutoff value, cases were interpreted as positive for polysomy (gain) or monosomy (deletion), respectively.

\section{Statistical analysis}

Categorical variables are presented as numbers and percentages, and were compared using the Fisher exact test. Continuous variables are expressed as means with standard deviations, and were compared using the Mann-Whitney U test. All statistical analyses were conducted using SPSS version 20.0 (IBM Corp. Armonk, NY, USA), and p-values $<0.05$ were considered statistically significant.

\section{RESULTS}

\section{Patient demographic and clinicopathological characteristics}

The demographic and clinicopathological characteristics of the study population are detailed in Table 2 . The mean age of the participants was 65.1 years, and 43 of them (66.2\%) were male. Pancreaticoduodenectomy was the most common treatment method, with distal pancreatectomy the second most common. Carbohydrate antigen 19-9 (CA19-9) levels were significantly higher in the PDAC group than the IPMN group. Pathologically, most patients with PDAC had T3 disease (44/48, 91.7\%) and lymph node metastases (37/48, 77.1\%). However, there were no statistically significant differences between the PDAC and invasive IPMN in pT stage $(p=0.080), p N$ stage $(p=0.173)$, angiolymphatic invasion $(p=0.413)$, perineural

Table 1. Cutoff Values for Each FISH Probe

\begin{tabular}{lccc}
\hline \multirow{2}{*}{ Probe } & \multicolumn{3}{c}{ Cutoff value (\%) } \\
\cline { 2 - 4 } & Monosomy & Polysomy* $^{*}$ & Aneuploidy $^{\dagger}$ \\
\hline 7q22/7q36 & 1.0 & 0.0 & 1.0 \\
$17 p 13$ & 1.0 & 0.0 & 1.0 \\
$18 q 21$ & 2.0 & 1.0 & 3.0 \\
$20 q 12$ & 1.0 & 0.0 & 1.0 \\
$21 q 22$ & 2.0 & 1.0 & 3.0 \\
CEP18 & 3.0 & 1.0 & 4.0 \\
\hline
\end{tabular}

FISH, fluorescence in situ hybridization; CEP18, chromosome enumeration probe 18 .

*Trisomy cutoff+tetrasomy cutoff; ${ }^{\dagger}$ Monosomy cutoff+polysomy cutoff. 
Table 2. Patient Demographic and Clinicopathologic Data

\begin{tabular}{|c|c|c|c|c|}
\hline Characteristic & All $(n=65)$ & $\operatorname{PDAC}(\mathrm{n}=48)$ & IPMN ( $\mathrm{n}=17)$ & p-value \\
\hline Age, yr & $65.1 \pm 9.7$ & $65.9 \pm 8.6$ & $63.1 \pm 12.5$ & 0.617 \\
\hline Male sex & $43(66.2)$ & $31(64.6)$ & $12(70.6)$ & 0.653 \\
\hline Types of operation & & & & 0.136 \\
\hline PD & $38(58.5)$ & $29(60.4)$ & $9(52.9)$ & \\
\hline Distal pancreatectomy & $20(30.8)$ & $16(33.3)$ & $4(23.5)$ & \\
\hline Other* & 7 (10.8) & $3(6.2)$ & $4(23.5)$ & \\
\hline CEA, ng/mL & $6.5 \pm 22.3$ & $7.9 \pm 25.9$ & $2.8 \pm 1.1$ & 0.662 \\
\hline CA19-9, U/mL & $1,055.0 \pm 2,786.1$ & $1,396.0 \pm 3,151.2$ & $32.1 \pm 31.9$ & $<0.001$ \\
\hline $\mathrm{pT}^{\dagger}$ & & & & 0.080 \\
\hline $\mathrm{T} 1$ & $2(3.7)$ & $1(2.1)$ & $1(16.7)$ & \\
\hline T3 & $48(88.9)$ & 44 (91.7) & $4(66.7)$ & \\
\hline $\mathrm{T} 4$ & $2(3.7)$ & $2(4.2)$ & 0 & \\
\hline $\mathrm{pN}^{\dagger}$ & & & & 0.173 \\
\hline No & $14(25.9)$ & $11(22.9)$ & $3(50.0)$ & \\
\hline N1 & $40(74.1)$ & $37(77.1)$ & $3(50.0)$ & \\
\hline Differentiation $^{\dagger}$ & & & & 0.010 \\
\hline Well/moderate/poor & $7 / 36 / 8$ & $7 / 32 / 8$ & $0 / 4 / 0$ & \\
\hline Unknown & 3 & 1 & 2 & \\
\hline Angiolymphatic invasion $(+)^{\dagger}$ & $26(48.1)$ & $22(45.8)$ & $4(66.7)$ & 0.413 \\
\hline Perineural invasion $(+)^{\dagger}$ & $44(81.5)$ & $41(85.4)$ & $3(50.0)$ & 0.070 \\
\hline Venous invasion $(+)^{\dagger}$ & $31(57.4)$ & $28(58.3)$ & $3(50.0)$ & 1.000 \\
\hline
\end{tabular}

Data are presented as mean \pm SD or number (\%).

PDAC, pancreatic ductal adenocarcinoma; IPMN, intraductal papillary mucinous neoplasm; PD, pancreaticoduodenectomy; CEA, carcinoembryonic antigen; CA19-9, carbohydrate antigen 19-9.

*Total pancreatectomy $(n=4)$, subtotal pancreatectomy $(n=2)$, central pancreatectomy $(n=1)$; ${ }^{\dagger}$ The reported pathologic data and statistical analysis of IPMN patients refer to only those with invasive IPMN $(n=6)$.

invasion ( $\mathrm{p}=0.070)$, and venous invasion $(\mathrm{p}=1.000)$.

\section{FISH analysis and comparison of PDAC and IPMN}

At least two chromosome alterations were detected in all patients with either PDAC or IPMN using standard cutoff values (Table 1). For the PDAC group, 17p deletion was the most frequently detected alteration (46/48, 95.8\%), followed by $18 \mathrm{q}$ deletion (40/48, 83.3\%), CEP18 deletion (39/48, 81.2\%), $20 q$ gain $(39 / 48,81.2 \%), 21 q$ deletion $(37 / 48,77.1 \%)$, and $7 q$ gain $(34 / 48,70.8 \%$ ) (Table 3$)$. For the IPMN group, $17 p$ deletion $(16 / 17,94.1 \%)$ and CEP18 deletion (16/17, 94.1\%) were also the most frequently detected alterations, followed by 21q deletion, 20q gain, $18 q$ deletion, and $7 q$ gain. CEP18 gain was significantly more frequent in the PDAC group than the IPMN group (26/48 vs 4/17, p=0.029), and the frequency of $18 \mathrm{q}$ deletion was marginally significantly different between the two groups (40/48 vs 10/17, p=0.051). The patterns of chromosomal alteration were similar between invasive and noninvasive IPMN, and there were no statistical differences between the two groups in the chromosomal alterations detected by each probe.
The details of frequent chromosomal alterations identified in invasive versus noninvasive IPMN were as follows: $7 q$ gain (4/6 vs $6 / 11, p=1.000$ ), $17 p$ deletion ( $6 / 6$ vs $10 / 11, p=1.000$ ), $18 q$ deletion ( $3 / 6$ vs $7 / 11, p=0.644$ ), 20q gain ( $4 / 6$ vs $7 / 11, p=1.000$ ), 21q deletion (6/6 vs 6/11, p=0.102), and CEP18 deletion (6/6 vs $10 / 11, \mathrm{p}=1.000$ )

There were statistically significant differences between the PDAC and IPMN groups in the proportion of cells with $17 \mathrm{p}$ deletion $(32.2 \pm 29.3$ vs $16.9 \pm 20.8, p=0.019)$ and $18 \mathrm{q}$ deletion $(27.7 \pm 30.2$ vs $7.0 \pm 13.5, p=0.004)$ (Fig. 2). For the other probes, there were no statistically significant differences in the chromosomal alteration rates between the PDAC group and IPMN group.

Modification of the cutoff value (percentage of cells positive for a chromosome alteration required for a positive score) resulted in statistically significant differences in mean alteration rates between the PDAC and IPMN groups for some probes as follows: $17 p$ deletion with cutoff values of $10.0 \%(36 / 48$ vs $7 / 17, p=0.011)$ and $20.0 \%$ (27/48 vs $3 / 17, p=0.006) ; 18 q$ deletion with cutoff values of $10.0 \%$ (29/48 vs $2 / 17, \mathrm{p}=0.001$ ) 
Table 3. FISH Analysis Results According to Pathologic Diagnosis

\begin{tabular}{|c|c|c|c|}
\hline $\begin{array}{l}\text { Chromosomal } \\
\text { alteration }\end{array}$ & PDAC (n=48) & IPMN (n=17) & $\mathrm{p}$-value \\
\hline \multicolumn{4}{|l|}{$7 q 22 / 7 q 36$} \\
\hline Monosomy & $14(29.2)$ & $3(17.6)$ & 0.523 \\
\hline Polysomy & $34(70.8)$ & $10(58.8)$ & 0.363 \\
\hline Aneuploidy & 38 (79.2) & $11(64.7)$ & 0.326 \\
\hline \multicolumn{4}{|l|}{ 17p13 } \\
\hline Monosomy & $46(95.8)$ & $16(94.1)$ & 1.000 \\
\hline Polysomy & $10(20.8)$ & $3(17.6)$ & 1.000 \\
\hline Aneuploidy & 44 (91.7) & $16(94.1)$ & 1.000 \\
\hline \multicolumn{4}{|l|}{$18 q 21$} \\
\hline Monosomy & 40 (83.3) & $10(58.8)$ & 0.051 \\
\hline Polysomy & $10(20.8)$ & 3 (17.6) & 1.000 \\
\hline Aneuploidy & 39 (81.2) & $10(58.8)$ & 0.100 \\
\hline \multicolumn{4}{|l|}{ 20q12 } \\
\hline Monosomy & 27 (56.2) & $10(58.8)$ & 0.854 \\
\hline Polysomy & 39 (81.2) & $11(64.7)$ & 0.191 \\
\hline Aneuploidy & 44 (91.7) & 15 (88.2) & 0.648 \\
\hline \multicolumn{4}{|l|}{$21 q 22$} \\
\hline Monosomy & 37 (77.1) & 12 (70.6) & 0.744 \\
\hline Polysomy & $17(35.4)$ & $8(47.1)$ & 0.397 \\
\hline Aneuploidy & 40 (83.3) & $14(82.4)$ & 1.000 \\
\hline \multicolumn{4}{|l|}{ CEP18 } \\
\hline Monosomy & 39 (81.2) & $16(94.1)$ & 0.270 \\
\hline Polysomy & $26(54.2)$ & $4(23.5)$ & 0.029 \\
\hline Aneuploidy & 47 (97.9) & $16(94.1)$ & 0.458 \\
\hline
\end{tabular}

Data are presented as number (\%).

FISH, fluorescence in situ hybridization; PDAC, pancreatic ductal adenocarcinoma; IPMN, intraductal papillary mucinous neoplasm; CEP18, chromosome enumeration probe 18.

and 20.0\% (23/48 vs $1 / 17, \mathrm{p}=0.002)$; and CEP18 gain with a cutoff value of 5.0\% (18/48 vs $2 / 17, \mathrm{p}=0.048)$.

\section{Associations of clinicopathologic features and results of FISH analysis}

Analysis of relationships between the commonly identified chromosomal alterations and clinicopathological factors demonstrated that CEP18 gain was significantly more frequent in older patients $(21 / 28$ vs $7 / 20, p=0.024)$ and those with lymph node metastasis (23/37 vs 3/11, p=0.041) (Table 4); however, no other probes exhibited any significant associations with clinicopathological factors.

For patients with PDAC, overall 1- and 2-year survival rates were $67.2 \%$ and 61.6\%, respectively. During follow-up (median, 15.5 months; range, 0 to 26 months), recurrence was diagnosed in 26 of 48 patients (54.2\%). The majority of recurrence was diagnosed within 12 months (21/26, 80.8\%), and the median time to recurrence was 5.0 months. Patients with recurrence had comparable carcinoembryonic antigen and CA19-9 levels and pathologic findings, including $\mathrm{T}$ stage, $\mathrm{N}$ stage, differentiation, angiolymphatic invasion, perineural invasion, and venous invasion, to those without recurrence (Table 5). There were also no significant differences between patients with recurrence and without recurrence in $7 q$ gain, $17 p$ deletion, 18q deletion, CEP18 deletion, 20q gain, 21q deletion, or CEP18 gain.

\section{Preoperative diagnosis of PDAC}

To clarify whether the investigated chromosomal alterations were useful for discriminating between PDAC and IPMN, we evaluated the diagnostic accuracies of various patterns of chromosomal alterations (Table 6). Analysis including the five common chromosomal aberrations in the PDAC group (7q gain, $17 p$ deletion, $18 \mathrm{q}$ deletion, $20 \mathrm{q}$ gain, and $21 \mathrm{q}$ deletion) and CEP18 gain, which were significantly more frequent than in the IPMN group, identified a mean of $4.6 \pm 0.9$ (range, 2 to 6 ) chromosomal alterations in the PDAC group, with $3.7 \pm 1.1$ (range, 2 to 6 ) in the IPMN group ( $\mathrm{p}=0.004)$. Selection of single probes resulted in higher diagnostic accuracy, with values of 79.3\% (sensitivity, 87.8\%; specificity, 58.8\%; and relative risk, 2.5) for $17 \mathrm{p}$ deletion (cutoff value, $10.0 \%$ ), and it slightly increased to $80.0 \%$ when $17 p$ deletion (cutoff value, 10.0\%) combined with $18 q$ deletion (standard cutoff value: sensitivity, 97.9\%; specificity, 29.4\%; and relative risk, 4.8) or 18q deletion with a $10.0 \%$ cutoff value (sensitivity, 89.6\%; specificity, 52.9\%; and relative risk, 2.3).

When we analyzed the 25 PDAC patients who had preoperative histologic diagnosis, all six patients (100.0\%) who had not been diagnosed of malignancy showed positive results by FISH analysis whether the diagnostic criteria was set to the $17 \mathrm{p}$ deletion (cutoff value, 10.0\%) combined with 18q deletion (standard cutoff value), or $18 q$ deletion (cutoff value, 10.0\%). The 18 (94.7\%), and $16(84.2 \%)$ of 19 patients who had preoperative diagnosis of malignancy showed positive results when the diagnostic criteria was set to the $17 p$ deletion (cutoff value, 10.0\%) combined with $18 \mathrm{q}$ deletion (standard cutoff value), or $18 \mathrm{q}$ deletion (cutoff value, 10.0\%), respectively.

\section{DISCUSSION}

The results of the present study demonstrate that chromosomal alterations are very frequent in tumor samples from patients with both PDAC and IPMN. The chromosomal aberration patterns in IPMN were similar to those in PDAC, there were no significant differences in most probe sets, and comparisons of invasive and noninvasive IPMNs also demonstrated no significant differences between these groups. These results imply that similar early genetic alterations may be implicated in the development of both IPMN and PDAC, although they may be partly attributable to the fact that more than half of patients had severe dysplasia or invasive IPMN. Some previous cytogenetic studies support this assumption. 


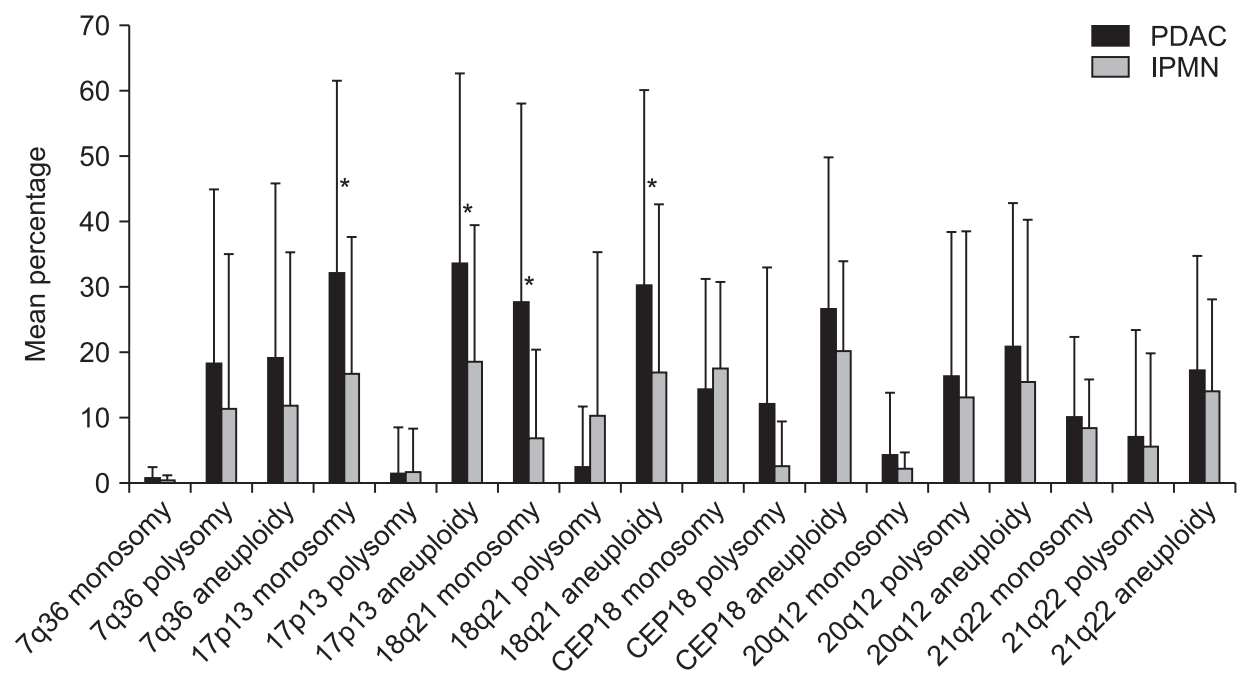

Fig. 2. Fluorescence in situ hybridization analysis results according to pathologic diagnosis. Genetic alteration rates in pancreatic ductal adenocarcinoma (PDAC) and intraductal papillary mucinous neoplasm (IPMN) are presented as the means and standard deviations. The rate of genetic alteration was higher in the PDAC group than in the IPMN group for almost all probes. The differences were significant for $17 \mathrm{p} 13$ monosomy (32.2 \pm 29.3 vs $16.9 \pm 20.8, p=0.019), 17 p 13$ aneuploidy (33.7 \pm 28.9 vs $18.7 \pm 20.7, p=0.030), 18 q 21$ monosomy (27.7 \pm 30.2 vs $7.0 \pm 13.5, p=0.004)$, and $18 \mathrm{q} 21$ aneuploidy (30.4 \pm 29.7 vs $17.0 \pm 25.6, \mathrm{p}=0.031)$.

CEP18, chromosome enumeration probe 18 . ${ }^{*} \mathrm{p}<0.05$.

Fujii et al. ${ }^{11}$ conducted PCR-based microsatellite analysis of 13 IPMN specimens and found frequent loss of heterozygosity at $6 q, 8 p, 9 p, 17 p$, and $18 q$ with ratios of $31 \%$ to $62 \%$. Fritz et al. ${ }^{10}$ investigated 20 IPMN specimens by microarray-based comparative genomic hybridization analysis and reported frequent loss of chromosomes 2, 4q, 5q, 6q, 8p, 10q, 11q, 13q, $15 q, 18 q$, and $22 q$ with ratios of $38.5 \%$ to $76.9 \%$, and gains of chromosomes 7 and $19 q$ in half of specimens from invasive IPMN or IPMN with severe dysplasia. Both studies identified chromosomal aberrations also frequently identified in PDAC., ${ }^{4,5}$ Nevertheless, the chromosomal changes in IPMN have not been fully elucidated and further studies are warranted, as most previous studies have been based on small numbers of tissue samples.

The present study identified that using a modified cutoff value of 10\%, deletions in 17p13 (TP53) and 18q21 (SMAD4/ $D P C 4)$ were significantly more frequent in PDAC than IPMN. Both the TP53 and SMAD4/DPC4 genes are well-known tumor suppressors reportedly inactivated in more than 50\% of PDACs. ${ }^{3}$ Previous studies of genetic or protein loss of TP53 and SMAD4 revealed rising incidence with increasing PanIN grade. ${ }^{25,26}$ The present study supports the previously proposed tumor progression model for PDAC, which postulates that genetic changes at these loci may be involved in the late steps of carcinogenesis. ${ }^{27}$ However, the difference of chromosomal alteration between the PDAC and IPMN might be due to the difference of pathway between PanIN- and IPMN-derived carcinogenesis. The deletion rates of chromosome $17 p$ and $18 q$ have been reported to range from $80 \%$ to $100 \%$, and $56 \%$ to $88 \%$ in PDAC or PanIN with high grade dysplasia, and from
$73 \%$ to $100 \%$, and $54 \%$ to $100 \%$ of IPMN with high grade dysplasia or invasion, respectively. ${ }^{12,13,21,28}$ However, some other studies reported that the deletion of $17 p$ and $18 q$ even in PanIN-1 in 87\% and 50\%, respectively, and the SMAD4/DPC4 gene was inactivated only 3\% of IPMN. ${ }^{13,29}$ There have been only a small number of studies concerning the chromosomal alteration of the precursor lesions of PDAC, and future studies are necessary to elucidate how it is involved at any stage of the carcinogenesis.

The present study also determined that CEP18 gain was significantly more frequent in PDAC; however, little is known about the significance of changes in chromosome 18 copy number, particularly gain, in pancreatic carcinogenesis. On the contrary, some previous cytogenetic studies reported consistent frequent loss of chromosome 18 in PDAC, which was also identified in the present study., ${ }^{4,8}$ Gain of chromosome 18 has been reported in lymphoproliferative diseases, including acute lymphocytic leukemia, multiple myeloma, and non-Hodgkin's lymphoma; however, its role in carcinogenesis and clinica significance has yet to be elucidated. ${ }^{30}$ For pancreatic disease, Miyabe et al. ${ }^{12}$ reported that polysomy 18 (CEP18) was significantly more frequent in invasive IPMN, and may be involved in malignant transformation of IPMN, along with polysomy 7 and P16/TP53 deletion. Further studies are necessary to clarify the significance in carcinogenesis and clinical impact of CEP18.

The present study did not reveal clear associations between chromosomal aberrations and clinicopathologic features, including disease recurrence and patient survival, other than for CEP18 gain, which was more frequent in older patients and those with lymph node metastasis. This may be because 


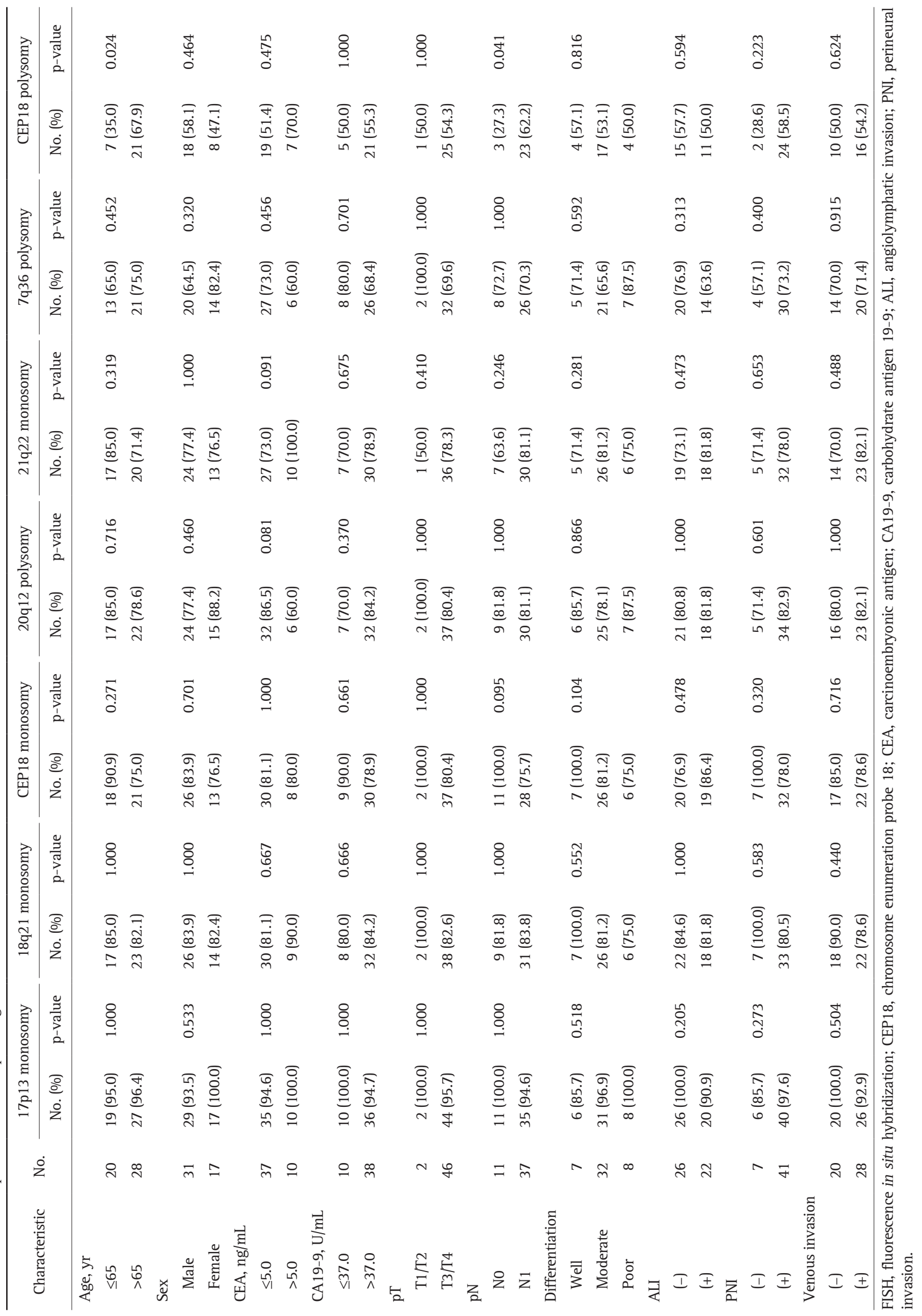


Table 5. Clinicopathological Differences and FISH Results According to Cancer Recurrence

\begin{tabular}{|c|c|c|c|c|}
\hline Characteristic & All $(n=48)$ & No recurrence $(n=22)$ & Recurrence $(\mathrm{n}=26)$ & $\mathrm{p}$-value \\
\hline Age, yr & $65.9 \pm 8.6$ & $66.4 \pm 6.3$ & $65.4 \pm 10.2$ & 0.868 \\
\hline Male sex & $31(64.6)$ & $15(68.2)$ & $16(61.5)$ & 0.632 \\
\hline Type of operation & & & & 0.867 \\
\hline PD & $29(60.4)$ & $14(63.6)$ & $15(57.7)$ & \\
\hline Distal pancreatectomy & 16 (33.3) & $7(31.8)$ & $9(34.6)$ & \\
\hline Other* & $3(6.3)$ & $1(4.5)$ & $2(7.7)$ & \\
\hline CEA, ng/mL & $7.9 \pm 25.9$ & $12.4 \pm 38.3$ & $4.2 \pm 5.5$ & 0.424 \\
\hline CA19-9, U/mL & $1,396.0 \pm 3,151.2$ & $1,682.5 \pm 3,577.5$ & $1,153.6 \pm 2,790.0$ & 0.521 \\
\hline pT & & & & 0.246 \\
\hline $\mathrm{T} 1$ & $1(2.1)$ & $1(4.5)$ & 0 & \\
\hline $\mathrm{T} 2$ & $1(2.1)$ & $1(4.5)$ & 0 & \\
\hline T3 & 44 (91.7) & 19 (86.4) & 25 (96.2) & \\
\hline $\mathrm{T} 4$ & $2(4.2)$ & $1(4.5)$ & $1(3.8)$ & \\
\hline $\mathrm{pN}$ & & & & 0.977 \\
\hline No & $11(22.9)$ & $5(22.7)$ & $6(23.1)$ & \\
\hline N1 & $37(77.1)$ & 17 (77.3) & 20 (76.9) & \\
\hline Differentiation & & & & 0.695 \\
\hline Well/moderate/poor & $7 / 32 / 8$ & $4 / 15 / 3$ & $3 / 17 / 5$ & \\
\hline Unknown & 1 & 0 & 1 & \\
\hline Angiolymphatic invasion (+) & $22(45.8)$ & $9(40.9)$ & $13(50.0)$ & 0.529 \\
\hline Perineural invasion $(+)$ & $41(81.5)$ & $19(86.4)$ & $22(84.6)$ & 1.000 \\
\hline Venous invasion (+) & $28(58.3)$ & $12(54.5)$ & $16(61.5)$ & 0.624 \\
\hline \multicolumn{5}{|l|}{ FISH analysis } \\
\hline 17p13 monosomy (+) & $46(95.8)$ & $22(100.0)$ & $24(92.3)$ & 0.493 \\
\hline $18 q 21$ monosomy (+) & 40 (83.3) & $21(95.5)$ & $19(73.1)$ & 0.055 \\
\hline CEP18 monosomy $(+)$ & $39(81.2)$ & $18(81.8)$ & $21(80.8)$ & 1.000 \\
\hline 20q12 polysomy (+) & $39(81.2)$ & $18(81.8)$ & $21(80.8)$ & 1.000 \\
\hline 21q22 monosomy (+) & 37 (77.1) & 17 (77.3) & 20 (76.9) & 0.977 \\
\hline 7q36 polysomy (+) & $34(70.8)$ & $15(68.2)$ & $19(73.1)$ & 0.710 \\
\hline CEP18 polysomy (+) & $26(54.2)$ & $13(59.1)$ & $13(50.0)$ & 0.529 \\
\hline
\end{tabular}

Data are presented as mean \pm SD or number (\%).

FISH, fluorescence in situ hybridization; PD, pancreaticoduodenectomy; CEA, carcinoembryonic antigen; CA19-9, carbohydrate antigen 19-9; CEP18, chromosome enumeration probe 18.

*Total pancreatectomy $(\mathrm{n}=1)$, subtotal pancreatectomy $(\mathrm{n}=2)$.

this study was performed on small patient populations with relatively short-term follow-up. Moreover, approximately $90 \%$ of patients had T3 disease and about 80\% had lymph node metastases, which could mask the effects of chromosomal alterations. However, there are some reports of a relationship between chromosomal alterations and clinicopathologic prognostic factors. Gutiérrez et al. ${ }^{31}$ reported that changes of chromosomes 7, 17q, 18q21, and 20 were significantly more frequent in advanced TNM stage tumors, and that numerical changes of chromosomes 4 and 9q34, together with gains of chromosome 8q24, were associated with reduced overall survival of patients. Stoecklein et al. ${ }^{32}$ reported that chromosome
17 ploidy level was negatively associated with disease free survival and overall survival.

Although EUS-guided cytology and core needle biopsy have been the primary tools for diagnosis of PDAC, they (particularly cytology) have been discredited because of low diagnostic sensitivity. The diagnostic yields of pancreatic EUS-guided fineneedle aspiration and core needle biopsy indicate sensitivities for these techniques of $54 \%$ to $96 \%$, and $71 \%$ to $99 \%$, respectively, and the present study showed similar sensitivity (76.0\%). ${ }^{14,15}$ The main limitation of cytology is false-negative results in patients with PDAC, which can be attributed to various factors, including difficulties in cytologic interpretation 
Table 6. Parameters for Pancreatic Ductal Adenocarcinoma Diagnosis Using FISH

\begin{tabular}{|c|c|c|c|c|}
\hline Diagnostic criteria & Sensitivity (\%) & Specificity (\%) & Accuracy (\%) & Relative risk \\
\hline All 6 probes $(+)$ & 15.2 & 100.0 & 38.1 & 1.4 \\
\hline$\geq 5$ Probes $(+)$ & 60.4 & 70.6 & 63.1 & 1.4 \\
\hline$\geq 4$ Probes $(+)$ & 89.6 & 41.2 & 76.9 & 1.9 \\
\hline$\geq 3$ Probes $(+)$ & 97.9 & 17.6 & 76.9 & 3.1 \\
\hline 17p13 (+) & 95.8 & 5.9 & 72.3 & 1.1 \\
\hline 18q21(+) & 83.3 & 41.2 & 72.3 & 1.5 \\
\hline $7 q 36(+)$ & 70.8 & 41.2 & 63.1 & 1.2 \\
\hline CEP18 (+) & 54.2 & 76.5 & 60.0 & 1.4 \\
\hline $20 q 12(+)$ & 81.3 & 35.3 & 69.2 & 1.3 \\
\hline $21 q 22(+)$ & 77.1 & 29.4 & 64.6 & 1.1 \\
\hline 17p13 $(10 \%)^{*}(+)$ & 87.8 & 58.8 & 79.3 & 2.5 \\
\hline 17p13 $(20 \%)^{\dagger}(+)$ & 56.3 & 82.4 & 63.1 & 1.5 \\
\hline 18q21 (10\%)* $(+)$ & 60.4 & 88.2 & 67.7 & 1.7 \\
\hline $18 \mathrm{q} 21(20 \%)^{\dagger}(+)$ & 47.9 & 94.1 & 60.0 & 1.6 \\
\hline 17p13 and 18q21 (+) & 79.2 & 47.1 & 70.8 & 1.5 \\
\hline 17p13 (10\%)" and 18q21 (+) & 60.4 & 70.6 & 63.1 & 1.4 \\
\hline 17p13 $(10 \%)^{*}$ or $18 q 21(+)$ & 97.9 & 29.4 & 80.0 & 4.8 \\
\hline $17 p 13(20 \%)^{\dagger}$ and $18 q 21(+)$ & 45.8 & 82.4 & 55.4 & 1.4 \\
\hline 17 p13 and 18q21 $(10 \%)^{*}(+)$ & 56.3 & 94.1 & 66.2 & 1.7 \\
\hline 17p13 and 18q21 $(20 \%)^{\dagger}(+)$ & 45.8 & 94.1 & 58.5 & 1.5 \\
\hline 17p13 (10\%)* and 18q21 (10\%)* $(+)$ & 47.9 & 94.1 & 60.0 & 1.6 \\
\hline 17 p13 $(10 \%)^{*}$ or $18 q 21(10 \%)^{*}(+)$ & 89.6 & 52.9 & 80.0 & 2.3 \\
\hline $17 p 13(10 \%)^{*}$ or $18 q 21(20 \%)^{\dagger}(+)$ & 85.4 & 58.8 & 78.5 & 2.1 \\
\hline 17p13 (10\%)* or CEP18 (+) & 89.6 & 35.3 & 75.4 & 1.8 \\
\hline 17p13, 18q21, and CEP18 (+) & 41.7 & 88.2 & 53.8 & 1.4 \\
\hline 17p13 (10\%)*, 18q21 (10\%)*, and CEP18 (+) & 22.9 & 100.0 & 43.1 & 1.5 \\
\hline 17p13 (10\%) $)^{*}$, 18q21 (20\%) $)^{\dagger}$, and CEP18 (+) & 16.7 & 100.0 & 38.5 & 1.4 \\
\hline 17p13 $(20 \%)^{\dagger}, 18 q 21(10 \%)^{*}$, and CEP18 (+) & 14.6 & 100.0 & 36.9 & 1.4 \\
\hline $17 \mathrm{p} 13(20 \%)^{\dagger}, 18 \mathrm{q} 21(20 \%)^{\dagger}$, and CEP18 (+) & 8.3 & 100.0 & 32.3 & 1.4 \\
\hline 17p13 (10\%)", 18q21 (10\%)", or CEP18 (+) & 93.8 & 29.4 & 76.9 & 2.1 \\
\hline
\end{tabular}

FISH, fluorescence in situ hybridization; 7q36, 7q36 polysomy; 17p13, 17p13 monosomy; 18q21, 18q21 polysomy; 20q12, 20q12 polysomy; 21q22, 21q22 monosomy; CEP, chromosome enumeration probe; CEP 18, CEP18 polysomy.

*Upper cutoff value, $10 \%$; ${ }^{\dagger}$ Upper cutoff value, $20 \%$.

of specimens with inflammatory cells, induced by adjacent chronic pancreatitis or recent instrumentation; paucicellular specimens, which harbor few or no malignant cells; and welldifferentiated carcinomas, which are difficult to discern. ${ }^{33}$ FISH can be used to analyze limited tissue material, including small biopsies, and samples from brushing or aspiration cytology, and has the ability to detect chromosomal alterations common in malignant tumors. This technique has been increasingly used in research and clinical practice for detection of pancreatobiliary malignancy in cytology specimens. ${ }^{17-20}$

The present study revealed the highest diagnostic accuracy for PDAC (80.0\%) of FISH tests positive for $17 \mathrm{p}$ deletion or $18 \mathrm{q}$ deletion, with a cutoff value of $10.0 \%$, and single probe detection of $17 \mathrm{p}$ deletion (cutoff value, 10.0\%) had a diagnostic accuracy of $79.3 \%$, with acceptable sensitivity (87.8\%) and specificity (58.8\%). We also found that these probe sets might be helpful in increasing the preoperative diagnostic accuracy for the patients who were not diagnosed with PDAC with conventional histologic examinations. Over decades, some studies have reported the clinical utility of UroVysion FISH using pancreatobiliary brushing specimens, revealing sensitivity significantly higher than that of conventional cytology for detection of malignancy. ${ }^{17-20}$ UroVysion FISH has a diagnostic sensitivity of $34 \%$ to $58 \%$, which is higher than that of routine cytology (8\% to 40\%); however, approximately half of patients with malignancy remain undiagnosed by FISH. ${ }^{16-20}$ However, 
only a few FISH studies of pancreatobiliary malignancies have used probes other than the UroVysion FISH probe set. Miyabe et al. ${ }^{12}$ reported that polysomy 7, polysomy 18, P16 deletion, and TP53 deletion were significantly more frequent in invasive IPMN, and that detection of polysomy 7 or TP53 deletion had potential value as diagnostic markers for invasive IPMN. Barr Fritcher et al. ${ }^{16}$ reported that the combination of the FISH probes, 1q21, 7p12, 8q24, and 9p21, identifies cancer cells with 93\% sensitivity and 100\% specificity, and has significantly higher sensitivity (64.7\%) than the UroVysion probes (45.9\%) or routine cytology analysis (18.8\%). The FISH probe sets used in the present study, which target genes associated with PDAC, showed acceptable diagnostic accuracy, and could be useful as an adjunct to conventional histopathologic examination.

This study has some limitations. First, as our sample size was relatively small and FISH analysis was performed with relatively few probe sets, the statistical power may be limited. This may account for the failure to elucidate any correlation between chromosomal alterations and clinicopathologic factors, including disease recurrence and patient survival. Second, this study compared chromosomal alterations of PDAC to those of IPMN. Because one of the aims of this study was to find out the useful FISH probe sets which could aid preoperative histologic diagnosis, we selected the probes directed to the chromosomes which alterations had been reported relatively frequent in PDAC to increase the diagnostic sensitivity, and set IPMN as a control group to identify the discrimination power of selected probes because IPMN might harbor similar chromosomal alterations to PDAC. However, the chromosomal alterations during the PanINderived carcinogenesis would be different from that from IPMNderived pathway. Because we experimented with cryopreserved tissue samples other than paraffin blocks, we could not harvest more premalignant tissue samples of PanIN and IPMN lesions with low to high grade dysplasia, which might make it possible to elucidate the differences of chromosomal alterations during the PanIN- and IPMN-derived pancreatic carcinogenesis. Nevertheless, we believe the commonality and the differences of chromosomal alteration between PDAC and IPMN identified in the present study could provide helpful information about the carcinogenesis of PDAC for conducting future studies. Lastly, this study was performed using tissue samples obtained by surgical resection, which harbored sufficient cells for analysis. This could have resulted in overestimation of diagnostic accuracy. Therefore, further studies are required to apply our findings in clinical practice using limited cytology specimens or small biopsies, and attempts to identify a more specific FISH probe set devoted to detection of chromosomal alterations typical of PDAC are warranted

In conclusion, chromosomal alterations were frequently identified in both PDACs and IPMNs. PDACs had 17p deletion, 18q deletion, CEP18 deletion, $20 q$ gain, $21 q$ deletion, and $7 q$ gain in more than $70 \%$ of patients, and IPMNs had a similar chromo- somal aberration pattern; however, IPMNs had a lower positive rate. Gain of chromosome 18 and deletions in $17 p$ and $18 q$ may be involved in the late steps of PDAC carcinogenesis. Although there were no clear clinicopathological associations with chromosomal alterations, deletions at chromosome $17 p$ and $18 q$ may represent excellent diagnostic markers for PDAC.

\section{CONFLICTS OF INTEREST}

No potential conflict of interest relevant to this article was reported.

\section{ACKNOWLEDGEMENTS}

This study was supported by the Collaborative Genome Program for Fostering New Post-Genome Industry of the National Research Foundation funded by the Ministry of Science and ICT (NRF-2017M3C9A5031597) and the Korean Health Technology R\&D Project, Ministry of Health \& Welfare (HI14C2640), Republic of Korea.

\section{AUTHOR CONTRIBUTIONS}

Data analysis and interpretation: C.S.L., K.I., W.K., J.R.K., Y.H. Data acquisition: K.I., Y.H. Drafting the manuscript: C.S.L. Critical revision of the manuscript, study supervision: D.S.L., S.W.K. J.Y.J. Study concept and design: J.Y.J.

\section{ORCID}

Chang-Sup Lim https://orcid.org/0000-0002-2349-9647

Kyongok Im https://orcid.org/0000-0002-2883-0183

Dong Soon Lee https://orcid.org/0000-0002-7085-9270

Wooil Kwon https://orcid.org/0000-0002-4827-7805

Jae Ri Kim https://orcid.org/0000-0001-6865-1350

Youngmin Han $\quad$ https://orcid.org/0000-0003-0456-7824

Sun-Whe Kim https://orcid.org/0000-0001-6315-6019

Jin-Young Jang https://orcid.org/0000-0003-3312-0503

\section{REFERENCES}

1. Rahib L, Smith BD, Aizenberg R, Rosenzweig AB, Fleshman JM, Matrisian LM. Projecting cancer incidence and deaths to 2030: the unexpected burden of thyroid, liver, and pancreas cancers in the United States. Cancer Res 2014;74:2913-2921.

2. Ansari D, Gustafsson A, Andersson R. Update on the management of pancreatic cancer: surgery is not enough. World J Gastroenterol 2015;21:3157-3165.

3. Notta F, Hahn SA, Real FX. A genetic roadmap of pancreatic cancer: still evolving. Gut 2017;66:2170-2178.

4. Griffin CA, Hruban RH, Morsberger LA, et al. Consistent chromosome abnormalities in adenocarcinoma of the pancreas. Cancer 
Res 1995;55:2394-2399.

5. Loukopoulos P, Shibata T, Katoh H, et al. Genome-wide arraybased comparative genomic hybridization analysis of pancreatic adenocarcinoma: identification of genetic indicators that predict patient outcome. Cancer Sci 2007;98:392-400.

6. Griffin CA, Hruban RH, Long PP, Morsberger LA, Douna-Issa F, Yeo CJ. Chromosome abnormalities in pancreatic adenocarcinoma. Genes Chromosomes Cancer 1994;9:93-100.

7. Schleger C, Arens N, Zentgraf H, Bleyl U, Verbeke C. Identification of frequent chromosomal aberrations in ductal adenocarcinoma of the pancreas by comparative genomic hybridization (CGH). J Pathol 2000;191:27-32.

8. Kowalski J, Morsberger LA, Blackford A, et al. Chromosomal abnormalities of adenocarcinoma of the pancreas: identifying early and late changes. Cancer Genet Cytogenet 2007;178:26-35.

9. Adsay NV, Dergham ST, Koppitch FC, et al. Utility of fluorescence in situ hybridization in pancreatic ductal adenocarcinoma. Pancreas 1999;18:111-116.

10. Fritz S, Fernandez-del Castillo C, Mino-Kenudson M, et al. Global genomic analysis of intraductal papillary mucinous neoplasms of the pancreas reveals significant molecular differences compared to ductal adenocarcinoma. Ann Surg 2009;249:440-447.

11. Fujii H, Inagaki M, Kasai S, et al. Genetic progression and heterogeneity in intraductal papillary-mucinous neoplasms of the pancreas. Am J Pathol 1997;151:1447-1454.

12. Miyabe K, Hori Y, Nakazawa T, et al. Locus/chromosome aberrations in intraductal papillary mucinous neoplasms analyzed by fluorescence in situ hybridization. Am J Surg Pathol 2015;39:512520.

13. Soldini D, Gugger M, Burckhardt E, Kappeler A, Laissue JA, Mazzucchelli L. Progressive genomic alterations in intraductal papillary mucinous tumours of the pancreas and morphologically similar lesions of the pancreatic ducts. J Pathol 2003;199:453461.

14. Lee YN, Moon JH, Kim HK, et al. Core biopsy needle versus standard aspiration needle for endoscopic ultrasound-guided sampling of solid pancreatic masses: a randomized parallel-group study. Endoscopy 2014;46:1056-1062.

15. Sur YK, Kim YC, Kim JK, Lee JH, Yoo BM, Kim YB. Comparison of ultrasound-guided core needle biopsy and endoscopic ultrasoundguided fine-needle aspiration for solid pancreatic lesions. J Ultrasound Med 2015;34:2163-2169.

16. Barr Fritcher EG, Voss JS, Brankley SM, et al. An optimized set of fluorescence in situ hybridization probes for detection of pancreatobiliary tract cancer in cytology brush samples. Gastroenterology 2015;149:1813-1824.

17. Fritcher EG, Kipp BR, Halling KC, et al. A multivariable model using advanced cytologic methods for the evaluation of indeterminate pancreatobiliary strictures. Gastroenterology 2009;136:21802186.

18. Smoczynski M, Jablonska A, Matyskiel A, et al. Routine brush cytology and fluorescence in situ hybridization for assessment of pancreatobiliary strictures. Gastrointest Endosc 2012;75:65-73.

19. Kipp BR, Stadheim LM, Halling SA, et al. A comparison of routine cytology and fluorescence in situ hybridization for the detection of malignant bile duct strictures. Am J Gastroenterol 2004;99:16751681.

20. Gonda TA, Glick MP, Sethi A, et al. Polysomy and p16 deletion by fluorescence in situ hybridization in the diagnosis of indeterminate biliary strictures. Gastrointest Endosc 2012;75:74-79.

21. Yamano M, Fujii H, Takagaki T, Kadowaki N, Watanabe H, Shirai T. Genetic progression and divergence in pancreatic carcinoma. Am J Pathol 2000;156:2123-2133.

22. Adsay V, Mino-Kenudson M, Furukawa T, et al. Pathologic evaluation and reporting of intraductal papillary mucinous neoplasms of the pancreas and other tumoral intraepithelial neoplasms of pancreatobiliary tract: recommendations of Verona Consensus Meeting. Ann Surg 2016;263:162-177.

23. American Joint Committee on Cancer (AJCC). Exocrine and endocrine pancreas. In: Edge SB, Byrd DR, Compton CC, Fritz AG, Greene FL, Trotti A, eds. AJCC Cancer Staging Manual. 7th ed. New York: Springer, 2010:241-249.

24. Ciolino AL, Tang ME, Bryant R. Statistical treatment of fluorescence in situ hybridization validation data to generate normal reference ranges using Excel functions. J Mol Diagn 2009;11:330333.

25. Lüttges J, Galehdari H, Bröcker V, et al. Allelic loss is often the first hit in the biallelic inactivation of the p53 and DPC4 genes during pancreatic carcinogenesis. Am J Pathol 2001;158:16771683

26. Wilentz RE, Iacobuzio-Donahue CA, Argani P, et al. Loss of expression of Dpc4 in pancreatic intraepithelial neoplasia: evidence that DPC4 inactivation occurs late in neoplastic progression. Cancer Res 2000;60:2002-2006.

27. Bardeesy N, DePinho RA. Pancreatic cancer biology and genetics. Nat Rev Cancer 2002;2:897-909.

28. Yoon YS, Lee DS, Min HC, et al. Analysis of molecular cytogenetic alteration of pancreatic cancer identified by fluorescent in situ hybridization (FISH) and its clinical significance. Korean J Hepatobiliary Pancreat Surg 2008;12:75-85.

29. Iacobuzio-Donahue CA, Klimstra DS, Adsay NV, et al. Dpc-4 protein is expressed in virtually all human intraductal papillary mucinous neoplasms of the pancreas: comparison with conventional ductal adenocarcinomas. Am J Pathol 2000;157:755-761.

30. Van Dyke D. +18 or trisomy 18 in lymphoproliferative disorders. Atlas Genet Cytogenet Oncol Haematol 2003;7:274-276.

31. Gutiérrez ML, Muñoz-Bellvis L, Sarasquete ME, et al. Altered interphase fluorescence in situ hybridization profiles of chromosomes 4, 8q24, and 9q34 in pancreatic ductal adenocarcinoma are associated with a poorer patient outcome. J Mol Diagn 2014;16:648659.

32. Stoecklein NH, Luebke AM, Erbersdobler A, et al. Copy number of chromosome 17 but not HER2 amplification predicts clinical outcome of patients with pancreatic ductal adenocarcinoma. J Clin 
520 Gut and Liver, Vol. 14, No. 4, July 2020

Oncol 2004;22:4737-4745.

33. Kipp BR, Barr Fritcher EG, Pettengill JE, Halling KC, Clayton AC. Improving the accuracy of pancreatobiliary tract cytology with fluorescence in situ hybridization: a molecular test with proven clinical success. Cancer Cytopathol 2013;121:610-619. 